\title{
SUCESIONES ESPECTRALES Y CURVATURA
}

\author{
Rolando Mosquera Ramírez \\ Universidad Nacional Mayor de San Marcos
}

\section{PRELIMINARES}

\section{INTRODUCCIÓN}

En [2] se construye la secuencia espectral $\left(E_{i}, \nabla_{i}\right)$. El objetivo de este trabajo es de obtener algunas relaciones entre las diferenciales de esta secuencia espectral, la curvatura y la curvatura seccional.

Nosotros primero construiremos el término $\left(E_{2}, \nabla_{2}\right)$ y el término general. Después expresaremos el borde en función de la curvatura, y luego aplicaremos los resultados para algunas variedades. Nosotros seguiremos [2], incluido las notaciones. Lo nuevo de este trabajo será la expresión del borde en función de la forma de curvatura y luego su relación con la curvatura seccional.

\section{SUCESIONES ESPECTRALES}

Sea $(E, \delta)$ un álgebra diferencial bigraduada. Sean.

$Z(E)$ los Cociclos de $E$

$B(E)$ los Cobordes de E

$A^{p, q}$ el conjunto de elementos de filtración $\geq p$ y grado total $p+q$

Sean

$$
\begin{aligned}
& Z^{P, q} \subset \frac{A^{P, q}}{A^{p+1, q-1}}, B^{p, q} \subset \frac{A^{p, q}}{A^{p+1, q-1}}, Z_{r}^{p, q} \subset \frac{A^{p, q}}{A^{p+1, q-1}}, B_{r}^{p, q} \subset \frac{A^{p, q}}{A^{p+1, q-1}}- \\
& (r \geq 0 \text { entero) tales que } \\
& Z^{p, q}=\left\{x \in \frac{A^{p, q}}{A^{p+1, q-1}} / \exists y \in x / y \in Z(E)\right\}
\end{aligned}
$$




$$
\begin{aligned}
& B^{p, q}=\left\{x \in \frac{A^{p, q}}{A^{p+1, q-1}} / \exists y \in x / y \in B(E)\right\} \\
& Z_{r}^{p, q}=\left\{x \in \frac{A^{p, q}}{A^{p+1, q-1}} / \exists y \in x / \text { filt } \delta y \geq p+r\right\} \\
& B_{r}^{p, q}=\left\{x \in \frac{A^{p, q}}{A^{p+1, q-1}} / \exists y \in x / y \text { es el coborde de un elemento de filt }>p-r\right\}
\end{aligned}
$$

Se tiene

$$
\begin{aligned}
& \subset Z_{r}^{p, q} \subset Z_{r-1}^{p, q} \subset \ldots \subset Z_{1}^{p, q} \subset Z_{0}^{p, q}=\frac{A^{p, q}}{A^{p+1, q-1}} \\
& 0=B_{0}^{p, q} \subset B_{1}^{p, q} \subset \ldots \subset B_{r}^{p, q} \subset \ldots \\
& \bigcap_{r} Z_{r}^{p, q}=Z^{p, q} \\
& \bigcup B_{r}^{p, q}=B^{p, q}
\end{aligned}
$$

La sucesión espectral es

$$
E_{2}^{p, q}=\frac{Z_{2}^{p, q}}{B_{2}^{p, q}}, \quad E_{3}^{p, q}=\frac{Z_{3}^{p, q}}{B_{3}^{p, q}}, \ldots
$$

Definición de la diferencial $\delta r$. Sea $x \in E_{r}^{p, q}$ y sea $x^{\prime} \in E_{r}^{p, q}$ un representante de $x$.

Por definición de $Z_{r}^{p, q}$ si $x^{\prime \prime} \in x^{\prime}$ filt $\delta x^{\prime \prime} \geq p+r$.

Sea $\overline{x^{\prime}} \in \frac{A^{p+r, q-r+1}}{A^{p+r+1, q-r}}$ la clase que contiene $\delta x^{\prime \prime}$. Como $\delta x^{\prime \prime}$ siendo un coborde es un cociclo, la clase $\overline{x^{\prime}}$ pertenece a los cociclos de $\frac{A^{p+r, q-r+1}}{A^{p+r+1, q-r}}$, es decir, $\overline{x^{\prime}} \in Z^{p+r, q-r+1}$ y de (3) $\bar{x}^{\prime} \in Z_{r}^{p+r, q-r+1}$. Sea $\overline{x^{\prime} \in} \frac{Z_{r}^{p+r, q-r+1}}{B_{r}^{p+r, q-r+1}}$ la clase de $\bar{x}^{\prime}$ en $\frac{Z_{r}^{p+r, q-r+1}}{B_{r}^{p+r, q-r+1}}$. Por definición $\delta_{r}^{p, q} x=\overline{x^{\prime}}$ Esto da las diferenciales de la secuencia espectral. 


\section{LA SUCESIÓN ESPECTRAL $\left(E_{i}, \nabla_{i}\right)$}

Sea $(E, i, \theta, R, \delta)$ una operación con conexión algebraica $x$ y curvatura $\chi$. Si se considera $\chi$ como una aplicación lineal homogénea de grado cero.

$$
\chi: E^{*} \rightarrow R_{i=0}^{2}
$$

$R_{i=0}$ siendo anticonmutativa se tiene

$$
\chi(x) \wedge \chi(y)=(-1)^{d^{\circ} \chi(x) d^{\circ} \chi(y)} \chi(y) \wedge \chi(x)=\chi(y) \wedge \chi(x)
$$

y $\chi$ se puede extender a un homomorfismo de álgebras graduadas

$$
\chi_{v}: V E * \rightarrow R_{i=0}
$$

La restricción de $\chi_{v}$ a las álgebras invariantes da un homomorfismo

$$
(\chi v)_{\theta=0}:(V E *)_{\theta=0} \rightarrow R_{i=0, \theta=0}
$$

Este homomorfismo verifica

$$
\delta^{\circ}\left(\chi_{v}\right)_{\theta=0}=0
$$

En efecto, $\nabla_{i=0}$ siendo una antiderivación, de la identidad de Bianchi $\nabla x=0$ se tiene

$$
\begin{gathered}
\nabla \circ_{i=0} \chi_{v}(x \vee y)=\nabla_{i=0}\left(\chi_{v}(x \vee y)\right)=\nabla_{i=0}(\chi(x) \wedge \chi(y))= \\
\nabla_{i=0} \chi x \wedge \chi y+(-1)^{2} \chi x \wedge \nabla_{i=0} \chi y=0
\end{gathered}
$$

Pero como la restricción de $\nabla_{i=0} \quad a \quad R_{i=0, \theta=0}$ coincide con $\delta$, la restricción de $\nabla_{i=0} \chi_{v} \quad a \quad(V E *)_{\theta=0}$ verifica

$$
\delta{ }^{\circ}\left(\chi_{v}\right)_{\theta=0}=0
$$


Sea $\tau: P \rightarrow(V E *)_{\theta=0}$ una transgresión en $W(E)_{\theta=0}$. Definimos una aplicación lineal homogénea de grado 1.

$$
\tau_{R}: P \rightarrow R_{i=0, \theta=0}
$$

por el diagrama

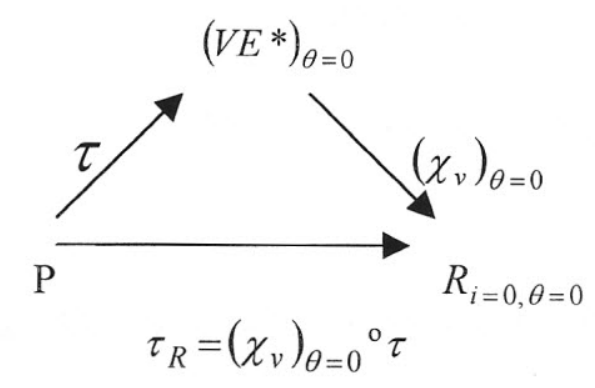

$$
\tau_{R}=\left(\chi_{v}\right)_{\theta=0}^{\circ} \tau
$$

$\operatorname{Como} \delta^{\circ}\left(\chi_{v}\right)_{\theta=0}=0$

$$
\delta^{\circ} \tau_{R}=0
$$

y se obtiene la $(P, \delta)$ - álgebra $\left(R_{i=0, \theta=0}, \delta ; \tau_{R}\right)$ que se llama la $(P, \delta)$ - álgebra asociada a la operación por medio de la conexión $\chi$ y la transgresión $\tau$. El complejo de Koszul de ésta álgebra es $\left(R_{i=0, \theta=0} \otimes \wedge P, \nabla\right) \operatorname{con} \nabla=\delta \otimes i+\nabla \tau$, donde $\nabla_{\tau} \quad$ es la "diferencial de transgresión" definida por

$$
\begin{gathered}
\nabla_{\tau}(a \otimes 1)=0 \\
\nabla_{\tau}\left(a \otimes x_{1} \wedge \cdots \wedge x_{2 k-1}\right)=(-1)^{p} \sum_{i=1}^{2 k-1}(-1)^{i-1} a \wedge \tau_{R}\left(x_{i}\right) \otimes x_{1} \wedge \cdots \wedge \hat{x}_{i} \wedge \cdots \wedge x_{2 k-1} \\
a \in R_{i=0, \theta=0}^{p}, x_{i} \in P
\end{gathered}
$$

$\nabla$ siendo una diferencial se tiene el álgebra de cohomología $H\left(R_{i=0, \theta=0} \otimes \wedge P, \nabla\right)$ de la $(P, \delta)$ - algebra $\left(R_{i=0 . \theta=0} \otimes \wedge P, \delta ; \tau_{R}\right)$. 
Si se da a $R_{i=0, \theta=0} \otimes \wedge P$ la bigraduación

$$
\left(R_{i=0, \theta=0} \otimes \wedge P\right)^{p, q} R_{i=0, \theta=0}^{p} \otimes(\wedge P)^{q}
$$

$\nabla$ es la suma de dos diferenciales: $\delta \otimes i$ de bigrado $(1,0)$ y $\nabla_{\tau}$ de bigrado $[2,-1)$. La filtración por el grado base

$$
F^{p}\left(R_{i=0, \theta=0} \otimes \wedge P\right)=\sum_{u \geq p} R_{i=0, \theta=0}^{u} \otimes \wedge P
$$

de la sucesión espectral $\left(E_{i}, \nabla_{i}\right)$ del álgebra diferencial bigraduada $\left(R_{i=0, \theta=0}^{p} \otimes(\wedge P)^{q}, \nabla\right)$.

\section{LA SUCESIÓN ESPECTRAL $\left(E_{i}, \nabla_{i}\right)$}

El objetivo de este capítulo es estudiar algunas relaciones entre las diferenciales de la sucesión espectral $\left(E_{i}, \nabla_{i}\right)$ y la curvatura.

\section{EL TÉRMINO $\left(E_{2}, \nabla_{2}\right)$}

Nosotros utilizamos las notaciones de 1.1.

$$
\begin{aligned}
& E=R_{i=0, \theta=0} \otimes \wedge P \quad, \quad \delta=\nabla \\
& A^{p, q}=\left\{R_{i=0, \theta=0}^{p} \otimes(\wedge p)^{q}+R_{i=0, \theta=0}^{p+1} \otimes(\wedge p)^{q-1}+\cdots+R_{i=0, \theta=0}^{p+q} \otimes 1\right. \\
& Z^{p, q}, B^{p, q}, Z_{r}^{p, q}, B_{r}^{p, q}(r \geq 0) \quad \text { contenidos } \quad \text { en } \quad \begin{array}{c}
A^{p, q} \\
A^{p, q, q-1}
\end{array} \text { tales que } \\
& Z^{p, q}=\left\{x \in \begin{array}{c}
A^{p, q} \\
A^{p+1, q-1}
\end{array} / \exists y \in x / y \in Z\left(R_{i=0, \theta=0} \otimes \wedge P\right)\right\} \\
& B^{p, q}=\left\{x \in \begin{array}{c}
A^{p, q} \\
A^{p+1, q-1}
\end{array} / \exists y \in x / y \in B\left(R_{i=0, \theta=0} \otimes \wedge P\right)\right\} \\
& Z_{r}^{p, q}=\left\{x \in \begin{array}{c}
A^{p, q} \\
A^{p+1, q-1}
\end{array} / \exists y \in x / \text { filt } \nabla y \geq p+r\right\}
\end{aligned}
$$


$B_{r}^{p, q}=\left\{x \in \frac{A^{p, q}}{A^{p+1, q-1}} / \exists y \in x / y\right.$ es el coborde de un elmento de filtración $\left.>p-r\right\}$

\section{Teorema}

i) $E_{2}^{p, q}=H^{p}\left(R_{i=0, \theta=0}\right) \otimes(\wedge p)^{q}$

ii) $\nabla_{2}=\left.\nabla\right|_{p_{1}}$

\section{Demostración}

i) Nosotros tenemos $E_{2}^{p, q}=\frac{Z_{2}^{p, q}}{B_{2}^{p, q}}$. Vamos a construir $Z_{2}^{p, q} \quad y \quad B{ }_{2}^{p, q}$.

Por definición tenemos

si $x \in Z_{2}^{p, q}$, un representante de $x$ se puede escribir como un elemento

$$
\begin{gathered}
x^{\prime}=a^{p} \otimes x_{1} \wedge \cdots \wedge x_{2 k-1} \in R_{i=0, \theta=0}^{p} \otimes(\wedge p)^{q} \\
a^{p} \in R_{i=0, \theta=0}^{p}, x_{1} \wedge \cdots \wedge x_{2 k-1} \in(\wedge p)^{q}, \quad 1+\cdots+2 k-1=q
\end{gathered}
$$

tal que la filtración de $\nabla x^{\prime}$ es igual o mayor que $p+2$.

El coborde de $x^{\prime}$ es

$$
\begin{gathered}
\nabla x^{\prime}=\nabla\left(a^{p} \otimes x_{1} \wedge \cdots \wedge x_{2 k-1}\right)=\left(\delta \otimes i+\nabla_{\tau}\right)\left(a^{p} \otimes x_{1} \wedge \cdots \wedge x_{2 k-1}\right)= \\
(\delta \otimes i)\left(a^{p} \otimes x_{1} \wedge \cdots \wedge x_{2 k-1}\right)+\nabla_{\tau}\left(a^{p} \otimes x_{1} \wedge \cdots \wedge x_{2 k-1}\right)= \\
\delta a^{p} \otimes x_{1} \wedge \cdots \wedge x_{2 k-1}+(-1)^{p} \sum_{i=1}^{2 k-1}(-1)^{i-1} a^{p} \wedge \tau_{R}\left(x_{i}\right) \otimes x_{1} \wedge \cdots \wedge x_{i} \cdots \wedge x_{2 k-1}= \\
\delta a^{p} \otimes x_{1} \wedge \cdots \wedge x_{2 k-1}+(-1)^{p}\left(a^{p} \wedge \tau_{R}\left(x_{1}\right) \otimes x_{3} \wedge \cdots \wedge x_{2 k-1}+\cdots\right. \\
\left.+a^{p} \wedge \tau_{R}\left(x_{2 k-1}\right) \otimes x_{1} \wedge \cdots \wedge x_{2 k-3}\right)
\end{gathered}
$$


Como la filtración de $\nabla x^{\prime}$ es mayor o igual a $p+2$ y la filtración de $\delta a^{p} \otimes x_{1} \wedge \cdots \wedge x_{2 k-1}$ es igual a $p+$

$$
\delta a^{p} \otimes x_{1} \wedge \cdots \wedge x_{2 k-1}=0
$$

Esto implica

$$
\delta a^{p}=0
$$

es decir $a^{p}$ es un cociclo de $R_{i=0, \theta=0}^{p}$ y se puede escribir

$$
\begin{gathered}
x^{\prime}=z^{p} \otimes x_{1} \wedge \cdots \wedge x_{2 k-1} \\
z^{p} \in Z^{p}\left(R_{i=0, \theta=0}\right), x_{1} \wedge \cdots \wedge x_{2 k-1} \in(\wedge p)^{q}
\end{gathered}
$$

donde $Z^{p}\left(R_{i=0, \theta=0}\right)$ representa los p-cociclos de $R_{i=0, \theta=0}$.

Esto da

$$
Z_{2}^{p, q}=Z^{p}\left(R_{i=0, \theta=0}\right) \otimes(\wedge P)^{q}
$$

De una manera análoga

$$
B_{2}^{p, q} \subset \frac{A^{p, q}}{A^{p+1, q-1}}=\frac{R_{i=0, \theta=0}^{p} \otimes(\wedge P)^{q}+R_{i=0, \theta=0}^{p+1} \otimes(\wedge P)^{q-1}+\cdots}{R_{i=0, \theta=0}^{p+1} \otimes(\wedge P)^{q-1}+R_{i=0, \theta=0}^{p+2} \otimes(\wedge P)^{q-2}+\cdots}
$$

Si $x \in B_{z}^{p, q}$, un representante de $x$ es un elemento

$$
x^{\prime}=a^{p} \otimes x_{1} \wedge \cdots \wedge x_{2 k-1} \in R_{i=0, \theta=0}^{p} \otimes(\wedge P)^{q}
$$

que es un coborde de un elemento de filtración p-1. Pero como el bigrado de $\delta \otimes i$ es $(1,0)$ y el bigrado de $\nabla_{\tau}$ es $(2,-1)$, la única posibilidad es que $x^{\prime}$ sea de la forma

$$
\begin{aligned}
x^{\prime}= & (\delta \otimes i)\left(c^{p-1} \otimes x_{1} \wedge \cdots \wedge x_{2 k-1}\right)= \\
= & \delta c^{p-1} \otimes x_{1} \wedge \cdots \wedge x_{2 k-1}, \\
& c^{p-1} \otimes x_{1} \wedge \cdots \wedge x_{2 k-1} \in R_{i=0, \theta=0}^{p-1} \otimes(\wedge P)^{q}
\end{aligned}
$$


ya que con $\nabla_{\tau}$ no existe ninguna posibilidad de obtener $x^{\prime}$ a partir de un elemento de filtración $p-1$. Esto da $a^{p}=\delta c^{p-1} \in B^{p}\left(R_{i=0, \theta=0}\right)$ donde $B^{p}\left(R_{i=0, \theta=0}\right)$ representa los p- cobordes de $R_{i=0, \theta=0} \quad \mathrm{y}$ se puede escribir $x^{\prime}=b^{p} \otimes x_{1} \wedge \cdots \wedge x_{2 k-1}, \quad b^{p} \in B^{p}\left(R_{i=0, \theta=0}\right)$, y así $B_{2}^{p, q}=B^{p}\left(R_{i=0, \theta=0}\right) \otimes(\wedge p)^{q}$

Finalmente se obtiene

$$
E_{2}^{p, q}=H^{p}\left(R_{i=0, \theta=0}\right) \otimes(\wedge p)^{q}
$$

ii) Sea $x \in E_{2}^{p, q}$. Como $Z_{2}^{p, q}=Z^{p}\left(R_{i=0, \theta=0}\right) \otimes(\wedge p)^{q}$ un representante $x^{\prime}$ de $x$ se puede escribir

$$
\begin{gathered}
x^{\prime}=z^{p} \otimes x_{1} \wedge \cdots \wedge x_{2 k-1} \\
z^{p} \in Z^{p}\left(R_{i=0, \theta=0}\right), x_{1} \wedge \cdots \wedge x_{2 k-1} \in(\wedge p)^{q}
\end{gathered}
$$

el coborde de $x^{\prime}$ es

$$
\begin{gathered}
\nabla x^{\prime}=\delta z p \otimes x_{1} \wedge \cdots \wedge x_{2 k-1}+(-1)^{p} z^{p} \wedge \tau_{R}\left(x_{1}\right) \otimes x_{3} \wedge \cdots \wedge x_{2 k-1} \\
+\cdots+z^{p} \wedge \tau_{R}\left(x_{2 k-1}\right) \otimes x_{1} \wedge \cdots \wedge x_{2 k-3}
\end{gathered}
$$

el término $\delta z^{p} \otimes x_{1} \wedge \cdots \wedge x_{2 k-1}=0$ porque $z^{p}$ es un cociclo de $R_{i=0, \theta=0 \text {. Sea }} \bar{x}^{\prime}$ la clase de $\nabla x^{\prime}$ en $\frac{A^{p+2, q-1}}{A^{p+3, q-2}}$, es decir $\left[\nabla x^{\prime}\right]=\left[z^{p} \wedge \tau_{R}\left(x_{1}\right) \otimes x_{3} \wedge \cdots \wedge x_{2 k-1}+\cdots\right]$

Como $\nabla x^{\prime}$ siendo un coborde es un cociclo la clase $\bar{x}^{\prime}$ pertenece a $Z^{p+2, q-1}$ y de (1.1.3) a $Z_{2}^{p+2, q-1} \cdot z^{p} \wedge \tau_{R}\left(x_{1}\right)$ pertenece a $Z^{p+2}\left(R_{i=0, \theta=0}\right)$ porque $\tau_{R}\left(x_{1}\right)$ siendo un cociclo de $R_{i=0, \theta=0}$. también lo es $z^{p} \wedge \tau_{R}\left(x_{1}\right)$, que es un cociclo de $R_{i=0, \theta=0}^{p+2}$ porque deg $\tau_{R}\left(x_{1}\right)=2$. Haciendo el cociente de $\overline{x^{\prime}}$ se obtiene $\overline{x^{\prime}} \in H^{p+2}\left(R_{i=0, \theta=0}\right) \otimes(\wedge P)^{q-1}$. Se obtiene así

$$
\nabla_{2} x=\overline{x^{\prime}}=\overline{z^{p}} \wedge \tau_{R}\left(x_{1}\right) \otimes(\wedge P)^{q-1}
$$


donde $z^{p} \wedge \tau_{R}\left(x_{1}\right)$ representa la clase de cohomología de $z^{p} \wedge \tau_{R}\left(x_{1}\right)$ en $H^{p+2}\left(R_{i=0, \theta=0}\right)$. De la expresión se tiene:

$\nabla_{2}$ es la restricción de $\nabla$ a $P_{1}, \quad \nabla_{2}=\left.\nabla\right|_{p 1}$.

\section{EL TERMINO GENERAL}

Se razonará por recurrencia. En $E_{2 l}$ tenemos el diagrama que explicita el borde

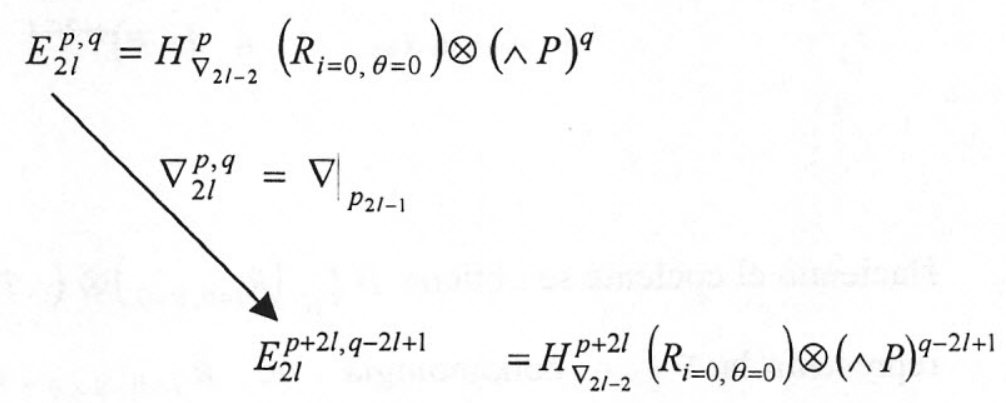

donde $H_{\nabla_{2 l-2}}\left(R_{i=0, \theta=0}\right)$ es la cohomología de $R_{i=0, \theta=0}$ con respecto al borde "multiplicación exterior de cociclos por $\nabla_{2 l-2}\left(x_{2 l-3}\right)=\chi_{v, \theta=0}\left(\tau\left(x_{2 l-3}\right)\right)=\tau_{R}\left(x_{2 l-3}\right)$ ".

Se va determinar $E_{2 l+2}^{p, q}$ como la $\nabla_{2 l}$ - cohomología de $E_{2 l}^{p, q}$. Si $x \in E_{2 l}^{p, q}$, un representante $x^{\prime}$ de $x$ es un elemento

$$
x^{\prime}=z^{p} \otimes x_{1} \wedge \cdots \wedge x_{2 k-1} \in Z_{2 l}^{p, q}
$$

tal que, si se define $\nabla_{0}\left(x^{\prime}\right)=\delta z^{p} \otimes x_{1} \wedge \cdots \wedge x_{2 k-1}\left(\nabla_{0}\left(z^{p}\right)=\delta z^{p}\right), x^{\prime}$ verifica

$$
\nabla_{0}\left(x^{\prime}\right)=\cdots=\nabla_{2 l-2}\left(x^{\prime}\right)=0
$$

condición que es equivalente a

$$
\nabla_{0}\left(z^{p}\right)=\cdots=\nabla_{2 l-2}\left(z^{p}\right)=0
$$

Entonces

$$
x^{\prime} \in Z_{\nabla_{2 l}}^{p, q}\left(R_{i=0, \theta=0} \otimes \wedge P\right) \Leftrightarrow \nabla_{0}\left(x^{\prime}\right)=\cdots=\nabla_{2 l}\left(x^{\prime}\right)=0
$$


condición que es equivalente a

$$
\nabla_{0}\left(z^{p}\right)=\cdots=\nabla_{2 l}\left(z^{p}\right)=0
$$

Lo mismo, sea y un elemento de $B_{2 l}^{p, q}$ y $y^{\prime}$ un representante de $y$.

\section{Entonces}

$$
\begin{aligned}
y^{\prime}= & \nabla_{2 l}\left(z^{p-2 l} \otimes x_{1} \wedge \cdots \wedge x_{2 k-1}\right) \\
= & z^{P-2 l} \wedge \tau_{R}\left(x_{2 l-1}\right) \otimes x_{1} \wedge \cdots \wedge \hat{x}_{2 l-1} \wedge \cdots \wedge x_{2 k-1}, \\
& x_{1} \wedge \cdots \wedge x_{2 k-1} \quad \in(\wedge P)^{q+2 l-1}
\end{aligned}
$$

Haciendo el cociente se obtiene $H_{\nabla_{21}}^{p}\left(R_{i=0, \theta=0}\right) \otimes(\wedge P)^{q}$, donde $H_{\nabla_{2 l}}^{p}\left(R_{i=0, \theta=0}\right)$ representa la $\nabla_{2 l}$ - cohomología de $R_{i=0, \theta=0}$, es decir, la cohomología de $R_{i=0, \theta=0}$ con respecto al borde "multiplicación exterior de cociclos por $\nabla_{2 l}\left(x_{2 l-1}\right)=\chi_{v, \theta=0}\left(\tau\left(x_{2 l-1}\right)\right)=\tau_{R}\left(x_{2 l-1}\right)$ ", borde en $R_{i=0, \theta=0}$, inducido por el borde $\nabla_{2 l}$ $\operatorname{de} E_{2 l}\left(R_{i=0, \theta=0} \otimes \wedge P\right)$.

Que esta multiplicación es un borde se verifica como en el caso de $\nabla_{2}$.

El borde $\nabla_{l+2}$. - Sea $x \in E_{2 l+2}^{p, q}$. Un representante $x^{\prime}$ de $x$ es un elemento $x^{\prime}=z P \otimes x_{1} \wedge \cdots \wedge x_{2-1} \in Z_{2 l+2}^{p, q}$ que verifica

$$
\nabla_{0}\left(x^{\prime}\right)=\cdots=\nabla_{2 l}\left(x^{\prime}\right)=0
$$

condición que es equivalente a

$$
\nabla_{0}\left(z^{p}\right)=\cdots=\nabla_{2 l}\left(z^{p}\right)=0
$$


El coborde de $x^{\prime}$ es

$$
\begin{aligned}
\nabla\left(x^{\prime}\right)= & \delta z^{p} \otimes x_{1} \wedge \cdots \wedge x_{2 k-1}+(-1)^{p} z^{p} \wedge \tau_{r}\left(x_{1}\right) \otimes x_{3} \wedge \cdots \wedge x_{2 k-1}+\cdots \\
& +(-1)^{p} z^{p} \wedge \tau_{R}\left(x_{2 l-1}\right) \otimes x_{1} \wedge \cdots \wedge \hat{x}_{2 l-1} \cdots \wedge x_{2 k-1}+ \\
& +(-1)^{p} z^{p} \wedge \tau_{R}\left(x_{2 l+1}\right) \otimes x_{1} \wedge \cdots \wedge \hat{x}_{2 l+1} \wedge \cdots \wedge x_{2 k-1}+\cdots \\
& +(-1)^{p} z^{p} \wedge \tau_{R}\left(x_{2 k-1}\right) \otimes x_{1} \wedge \cdots \wedge x_{2 k-3} \\
& =(-1)^{p} z^{p} \wedge \tau_{R}\left(x_{2 l+1}\right) \otimes x_{1} \wedge \cdots \wedge \hat{x}_{2 l+1} \wedge \cdots \wedge x_{2 k-1}+ \\
& +(-1)^{p} z^{p} \wedge \tau_{R}\left(x_{2 l+3}\right) \otimes x_{1} \wedge \cdots \wedge \hat{x}_{2 l+3} \wedge \cdots \wedge x_{2 k-1}+\cdots \\
& +(-1)^{p} z^{p} \wedge \tau_{R}\left(x_{2 k-1}\right) \otimes x_{1} \wedge \cdots \wedge x_{2 k-3}
\end{aligned}
$$

Sea $\overline{x^{\prime}}$ la clase de $\nabla x^{\prime}$ en $A^{p+2 l+2, q-2 l-1} / A^{p+2 l+3, q-2 l-2}$, es decir, $\bar{x}^{\prime}=\left[\nabla x^{\prime}\right] . \nabla x^{\prime}$ siendo un coborde es un cociclo y la clase $\overline{x^{\prime}}$ pertenece a $Z^{p+2 l+2, q-2 l-1}$ y de (1.1.3) pertenece a $Z_{2 l+2}^{p+2 l+2, q-2 l-1} \cdot z^{p} \wedge \tau_{R}\left(x_{2 l+1}\right)$ pertenece a $Z_{2 l+2}^{p+2 l+2}\left(R_{i=0, \theta=0}\right)$. Haciendo el cociente se obtiene $H_{\nabla_{2 l}}^{p+2 l+2}\left(R_{i=0, \theta=0}\right) \otimes(\wedge P)^{q-2 l-1}$.

Se obtiene así

\section{Teorema}

i) $E_{2 k}^{p, q}=H_{\nabla_{2 k-2}}^{p}\left(R_{i=0, \theta=0}\right) \otimes(\wedge P)^{p}$

ii) $\nabla_{2 k}=\left.\nabla\right|_{P_{2 k-1}}$

iii) $\nabla_{2 k}$ induce el borde en $R_{i=0, \theta=0}$ "multiplicación exterior de cociclos por $\nabla_{2 k}\left(x_{2 k-1}\right)=\chi_{v, \theta=0}\left(\tau\left(x_{2 k-1}\right)\right)=\tau_{R}\left(x_{2 k-1}\right) "$.

Observación.- Un elemento $\quad z^{p} \otimes x_{1} \wedge \cdots \wedge x_{2 k-1} \quad$ es un $\nabla_{2 l+2}$-cociclo $\left(\nabla_{2 l+2}-\right.$ coborde $)$ si y solamente si $z^{p}$ es un $\nabla_{2 l+2}$ cociclo $\left(\nabla_{2 l+2}-\right.$ coborde $)$.

\section{EXPRESIÓN DEL BORDE EN FUNCIÓN DE LA CURVATURA}

Se ha establecido que los bordes $\nabla_{2 r}$ en $E\left(R_{i=0, \theta=0} \otimes \wedge P\right)$ que son las restricciones de $\nabla$ a las componentes $P_{2 r-1}$ de $P$ inducen los bordes "multiplicación exterior de cociclos 
por $\nabla_{2 r}\left(x_{2 r-1}\right)$ en $R_{i=0, \theta=0}$ ", borde que continuamos denotando por $\nabla_{2 r}$. Vamos a probar que $\nabla_{2 r}$ en $R_{i=0, \theta=0}$ se puede expresar en función de la forma de curvatura.

Se tiene el diagrama que da el borde $\nabla$

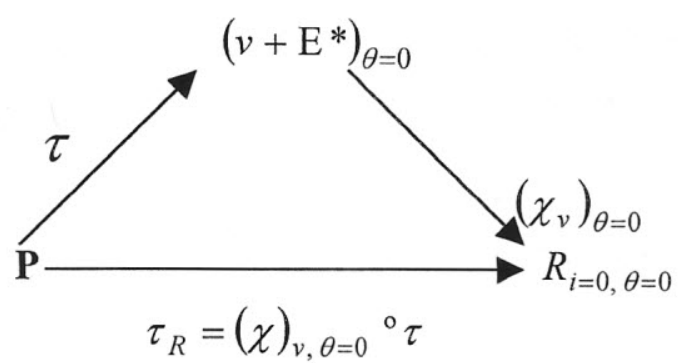

Sea $x_{1}, \cdots, x_{2 k-1}$ una base del espacio primitivo y sea

$$
\tau: P \rightarrow\left(V \mathrm{E}^{*}\right)_{\theta=0}
$$

una transgresión en $W(\mathrm{E})_{\theta=0}$ Nosotros tenemos:

Teorema $\boldsymbol{A}$ (P.K. Rashevskii [10]). Las imágenes por transgresión $\tau\left(x_{i}\right)$ de la base $\left(x_{i}\right), i=1, \cdots, 2 k-1$ de $P$ generan libremente $\left(V \mathrm{E}^{*}\right)_{\theta=0}$.

Teorema $\boldsymbol{B}$ (P.K. Rashevskii [10]). Todo elemento homogéneo diferente de cero $u \in\left(V \mathrm{E}^{*}\right)_{\theta=0}$ de grado $m(m=2,4, \ldots)$ es la imagen trangresiva de un elemento primitivo $x$ de grado $m-1$.

Lema.- Todo elemento $u \in\left(V \mathrm{E}^{*}\right)_{\theta=0}^{2}=\mathrm{E}_{\theta=0}^{*}$ es de la forma $u=\lambda \tau\left(x_{1}\right), \lambda \in R$.

Demostración.- En efecto del teorema A, $(\tau(x i)) i=1, \cdots, 2 k-1$ es una base de $\left(V \mathrm{E}^{*}\right)_{\theta=0}$. Si $u \in \mathrm{E}_{\theta=0}^{*}$ es evidente que en la expresión de $u$ no existen elementos de grado diferente de 2 , es decir $u=\lambda \tau\left(x_{1}\right), \lambda \in R$.

2.3.3. Teorema. $\nabla_{2 r}=\Omega \wedge \cdots \wedge \Omega \quad$ ( $r$ veces $)$ en $R_{i=0, \theta=0}$ salvo factores constantes, donde $\Omega$ es la forma de curvatura.

Demostración.- Nosotros tenemos el diagrama siguiente que explica el borde 


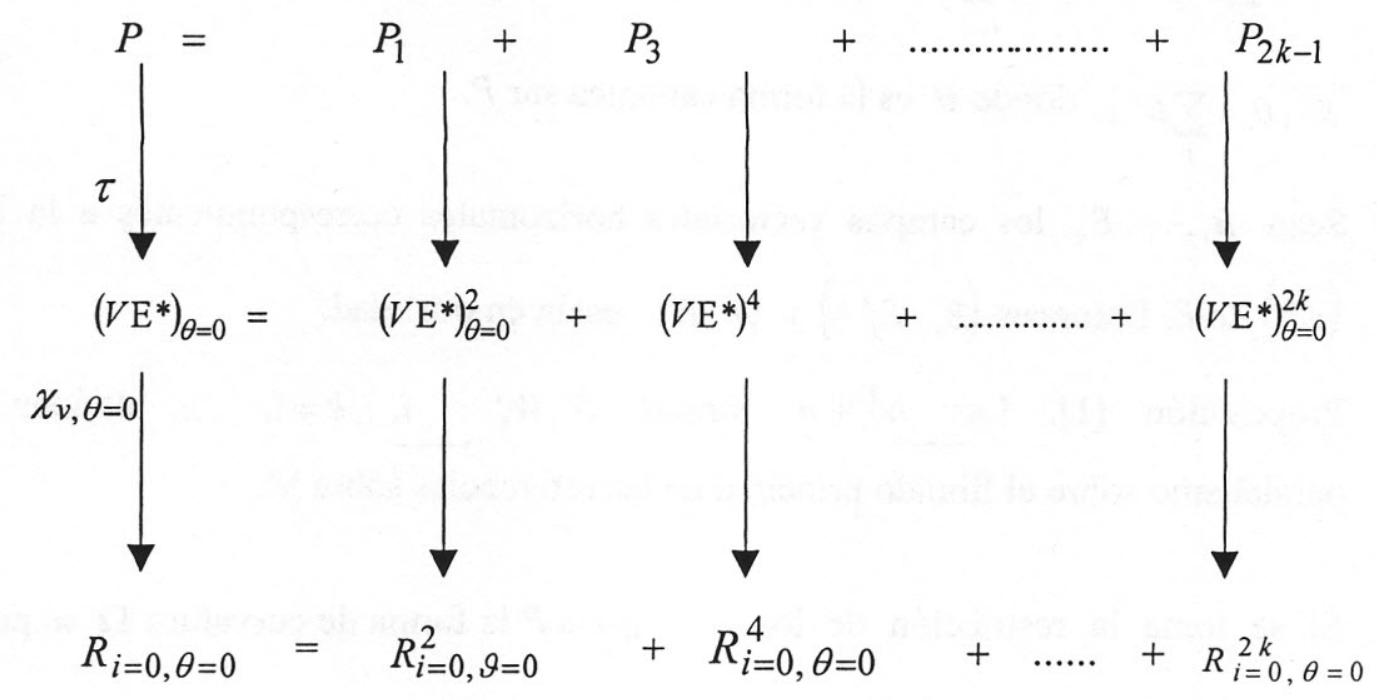

Si $u \in(V E *)_{\theta=0}^{2 r}$, como $\left(V E^{*}\right)_{\theta=0}$ siendo $\mathrm{E}$ reductiva es generado por $\mathrm{E}_{\theta=0}^{*}, u=y_{1} \vee \cdots \vee y_{r}, \quad y_{i} \in \mathrm{E}_{\theta=0}^{*}$. En vista del lema anterior $u=y_{1} \vee \cdots \vee y_{r}=\lambda_{1} \tau\left(x_{1}\right) \vee \cdots \vee \lambda_{r} \tau\left(x_{1}\right)$. Por el teorema B se puede suponer que $u$ es la imagen por transgresión de $x_{2 r-1} \in P_{2 r-1}$. Se tiene $\nabla_{2 r}\left(x_{2 r-1}\right)=\chi_{v, \theta=0}\left(\tau\left(x_{2 r-1}\right)\right)=\chi_{v, \theta=0}(u)=\lambda_{1} \cdots \lambda_{r} \chi\left(\tau\left(x_{1}\right)\right) \wedge \cdots \wedge \chi\left(\tau\left(x_{1}\right)\right)$.

Si se identifica $\chi\left(\tau\left(x_{1}\right)\right)=\Omega$ la forma de curvatura, se puede escribir

$$
\nabla_{2 r}=\Omega \wedge \cdots \wedge \Omega \quad(r \text { veces })
$$

salvo factores constantes.

\section{LA CURVATURA SECCIONAL Y LAS DIFERENCIALES DE LA SECUENCIA ESPECTRAL ASOCIADA A LA CONEXIÓN RIEMANNIANA}

\section{VARIEDADES RIEMANIANAS}

3.1.1. [1] Sea $M$ una variedad riemaniana de dimensión $n$ y sea $P$ el fibrado principal de las referencias ortonormales tangentes de grupo estructural el grupo ortogonal $O(n)$. Sea $W$ la conexión riemaniana y $\Omega$ la curvatura de $W$. Sea $\left(E_{i}^{j}\right)$ la base canónica de álgebra de Lie de $O(n)$. Nosotros tenemos 
$W=\sum_{i, j} W_{j}^{i} E_{i}^{j}, \Omega=\sum_{i, j} \Omega_{j}^{i} E_{i}^{j}, \quad y s i\left(e_{i}^{\prime}\right), i=1, \cdots, n$ es la base natural de $R^{n}, \theta=\sum_{i} \theta^{i} e_{i}^{\prime}$ donde $\theta$ es la forma canónica sur $P$.

Sean $B_{1}, \cdots, B_{n}$ los campos vectoriales horizontales correspondientes a la base $\left(E_{i}^{j}\right)$ de $E$. Entonces $\left(B_{i}, E_{i}^{j} *\right)$ y $\left\{\theta^{i}, W_{j}^{i}\right.$ están en dualidad.

Proposición [1]. Las $n^{2}+n$ formas $\theta^{i}, W_{k}^{i} \quad i, j, k=1, \cdots, n$ definen un paralelismo sobre el fibrado principal de las referencias sobre $\mathrm{M}$.

3.1.2. Si se toma la restricción de $\operatorname{los} \theta^{i} y W_{k}^{j}$ a $P$ la forma de curvatura $\Omega$ se puede expresar en términos de la $n_{1}$ - formas $\theta^{i}$.

Nosotros ponemos $\Omega_{j}^{i}=\sum_{k, l} \frac{1}{2} R_{j k l}^{i} \theta^{k} \wedge \theta^{l} \quad\left(R_{j k l}^{i}=-R_{j l k}^{i}\right)$ donde $R_{j k l}^{i}$ es el tensor de curvatura de la conexión riemaniana.

Para un arbitrario punto $u \in P \quad g_{i j}=\delta_{i j}$ y $R_{i j k l}=\sum_{s} g_{i s} R_{j k l}^{s}$ donde $g_{i j} \quad y \quad R_{i j k l}$ son el tensor métrico y el tensor riemaniano de curvatura.

3.1.3. Utilizando la definición de la curvatura seccional se tiene

$$
\begin{gathered}
\Omega_{j}^{i}=\sum_{k, l} \frac{1}{2} R_{j k l}^{i} \theta^{k} \wedge \theta^{l}=R_{j i j}^{i} \theta^{i} \wedge \theta^{j}+\sum_{\substack{k, l) \neq( \\
k \neq l)}} \frac{1}{2} R_{j k l}^{i} R_{j k l}^{i} \theta^{k} \wedge \theta^{l}= \\
R_{i j i j} \theta^{i} \wedge \theta^{j}+\sum_{\substack{k, l) \neq(i, j) \\
k \neq l)}} \frac{1}{2} R_{j k l}^{i} \theta^{k} \wedge \theta^{l}=K_{j}^{i} \theta^{i} \wedge \theta^{j}+\sum_{\substack{(k, l) \neq(i, j) \\
k \neq l)}} \frac{1}{2} R_{j k l}^{i} \theta^{k} \wedge \theta^{l}
\end{gathered}
$$

donde $k_{j}^{i}=k\left(e_{i}, e_{j}\right)$ es la curvatura seccional correspondiente al plano de base $e_{i}, e_{j}$ en el espacio tangente $T_{x} M, \pi(u)=x$, donde la base es $\left(e_{i}\right)_{i=1} \cdots, n$.

3.1.4. Además, de la definición del borde $\nabla_{2 r}=\left(\chi_{v, \theta=0}{ }^{\circ} \tau\right)_{P_{2 r-1}}, \quad r=1, \cdots$, como $\left(\chi_{\nu}\right)_{\theta=0}$ induce el homomorfismo de Weil, se ve que las imágenes pertenecen al álgebra característica de la fibración por definición, que en el caso del fibrado principal tangente es generado por las clases de Pontrjagin. 


\subsection{VARIEDADES CON CURVATURA CONSTANTE}

3.2.1. Como las clases de Pontrjagin son nulas para las variedades con curvatura constante, el álgebra característica será nula también y entonces $\nabla_{2 r}=0, r=1, \cdots, \mathrm{y}$ la secuencia espectral se detiene en el segundo término $E_{2}$.

\section{VARIEDADES CON CURVATURA VARIABLE}

\subsubsection{Teorema}

i) Los productos de las curvaturas seccionales $k_{j_{1}}^{i^{1}} k_{j_{2}}^{i^{2}} \cdots k_{j_{2} k}^{i^{2 k}}$ anulan las diferenciales de la secuencia espectral.

ii) La parte no nula proviene de los productos de la curvatura seccional por el tensor de curvatura donde se ha quitado la parte correspondiente a la curvatura seccional, o del tensor de curvatura por si mismo quitando la curvatura seccional.

Demostración. Tenemos la expresión de las clases de Pontrjagin en función de la curvatura

$$
g_{2 k}(\Omega)=\frac{1}{(2 \pi)^{2 k}(2 k) !} \sum_{i_{1} \cdots i_{2 k}}^{J_{1} \cdots J_{2 k}} \delta \quad \Omega_{j_{1}}^{i_{1}} \wedge \cdots \wedge \Omega_{j_{2 k}}^{i_{2 k}}
$$

donde se toma la suma sobre todos los subconjuntos ordenados $\left(i_{1}, \cdots, i_{2 k}\right)$ de $2 k$ elementos de $(1, \cdots, n)$ y toda las permutaciones $\left(j_{1}, \cdots, j_{2 k}\right)$ de $\left(i_{1}, \cdots, i_{2 k}\right)$ y $\delta_{i_{1} \ldots i_{2 k}}^{j_{1} \ldots j_{2 k}}$ es el signo de la permutación. Se tiene también la expresión (3.1.3.)

$$
\Omega_{j}^{i}=k_{j}^{i} \theta^{i} \wedge \theta^{j}+\frac{1}{2} \sum_{\substack{k, l) \neq(i, j) \\ k \neq l)}} R_{j k l}^{i} \theta^{k} \wedge \theta^{l}
$$

que da las componentes de la forma de curvatura. Haciendo el producto exterior se obtiene el resultado. El factor

$$
k_{j_{1}}^{i_{1}} k_{j_{2}}^{i_{2}} \cdots k_{j_{s}}^{i_{s}} \theta^{i_{1}} \wedge \theta^{j_{1}} \wedge \cdots \wedge \theta^{i_{s}} \wedge \theta^{j_{s}}
$$


es nulo porque en el conjunto $\left(i_{1}, j_{1}, \cdots, i_{s}, j_{s}\right)$ existen elementos repetidos que anulan el producto exterior y se puede decir que los productos $k_{j_{1}}^{i_{1}} k_{j_{2}}^{i_{2}} \cdots k_{j_{s}}^{i_{s}}$ anulan las diferenciales de la secuencia espectral.

\section{BIBLIOGRAFÍA}

1. KOBAYASHI AND K. NOMIZU, "Foundations of Differential Geometry I, II, 1969, intersciences.

2. W.GREUB, S. HALPERIN, R.Vanstone, "Connections, curvature and Cohomology I, II, III, Academic Press 1976.

3. R. GODEMENT, "Theórie des faisceaux, Hermann, Paris, 1958.

4. J. MILNOR AND J.C. Moore, "On the structure of Hopf Algebras, Ann. Of Math 81(2), 1965.

5. J.LEVAY, "L'anneau Spectrale et l'anneau filtré d'homologie d'un epace fibré dont la fibre est connexe" J. De Math, 1950, 9-139, 169-213.

6. H.CARTAN, a."Notions d'algebre differentielle; applications aux groupes de Lie et varietés ón ópere un groupe de Lie"; Colloque de Topologie (espaces fibrés), Bruxelles, 1950, Liége et Paris, 1951, 15-27. B."La transgression dans un groupe de Lie et dans un espace fibré principal" ibid 57-71.

7. M. ANDRÉ "Cohomologie des algébres differentielles ón ópere une algébre de Lie" Tohoku Math. J. 14(1962), 263-311.

8. A. BOREL, "Sur la cohomologie des espaces fibrés principanx et des espaces homogénes de groupes de Lie compacts", Ann. Of Math 57 (1953), 115-257.

9. D.B. Fuks, “The spectral Secuence of a fibering”, Russian Math Survey’s 21 (1966), 141-171.

10. P.K. RASHEVSKII, “The Real Cohomologie of Homogeneous Spaces”, Russian Math Survey's 24 (1), 1969 3eme, 23-95. 